\title{
Directed evolution of a ketone synthase \\ for efficient and highly selective functionalization of internal alkenes by accessing reactive carbocation intermediates
}

\author{
Sebastian Gergel, ${ }^{\text {a, }}$ Jordi Soler,,${ }^{\mathrm{b}, \#}$ Alina Klein, ${ }^{\mathrm{a}}$ Kai H. Schülke, ${ }^{a}$ Bernhard Hauer, ${ }^{\mathrm{c}}$ \\ Marc Garcia-Borràs, ${ }^{\mathrm{b}, *}$ Stephan C. Hammer ${ }^{\mathrm{a}, *}$

\footnotetext{
a Chair of Organic Chemistry and Biocatalysis, Faculty of Chemistry, Bielefeld University, Universitätsstraße 25, 33615 Bielefeld, Germany

b Institut de Química Computacional i Catàlisi and Departament de Química, Universitat de Girona, Carrer Maria Aurèlia Capmany 69, Girona 17003, Catalonia, Spain

c Department of Technical Biochemistry, Institute of Biochemistry and Technical Biochemistry, University of Stuttgart Allmandring 31, 70569 Stuttgart, Germany

\# both authors contributed equally

*Email: marc.garcia@udg.edu, stephan.hammer@uni-bielefeld.de
}

\begin{abstract}
The direct regioselective oxidation of internal alkenes to ketones could simplify synthetic routes and solve a longstanding challenge in synthesis. This reaction is of particular importance because ketones are predominant moieties in valuable products as well as crucial intermediates in synthesis. Here we report the directed evolution of a ketone synthase that oxidizes internal alkenes directly to ketones with several thousand turnovers. The evolved ketone synthase benefits from more than a dozen crucial mutations, most of them distal to the active site. Computational analysis reveals that all these mutations collaborate to facilitate the formation of a highly reactive carbocation intermediate by generating a confined, rigid and preorganized active site through an enhanced dynamical network. The evolved ketone synthase fully exploits a catalytic cycle that has largely eluded small molecule catalysis and consequently enables various challenging functionalization reactions of internal alkenes. This includes the first catalytic, enantioselective oxidation of internal alkenes to ketones, as well as the formal asymmetric hydration and hydroamination of unactivated internal alkenes in combination with other biocatalysts.
\end{abstract}




\section{Introduction}

Ketones are essential structural motifs in pharmaceuticals, agrochemicals, materials and natural products. They are also engaged as versatile intermediates in a wide range of reactions, including the synthesis of chiral alcohols and amines ${ }^{1}$. Thus, efficient catalytic methods for the production of ketones have long been sought. Selective ketone synthesis via simple aerobic oxidation of internal alkenes would represent a powerful addition to synthetic organic chemistry. This is because internal alkenes are easily accessible from petroleum and renewable resources as well as from well-established reactions such as olefin metathesis ${ }^{2}$ and carbonyl olefination ${ }^{3}$. Due to the lack of efficient and selective catalysts, the hydroboration-oxidation method using toxic and expensive boranes is still widely used ${ }^{4,5}$. Next to methods using stoichiometric reagents, various catalytic strategies to access ketones from internal alkenes have been developed (Fig. S1). In particular, attempts have been made to expand the well-studied Wacker-Tsuji oxidation from terminal towards internal alkenes ${ }^{6-9}$. In such palladium-catalyzed Wacker-type oxidations, however, internal alkenes are less reactive, which results in high catalyst loading and consequently low total turnover numbers (<20 TTN). Besides activity, selectivity is a major challenge in catalyst development (Fig. 1a). High regioselectivities in Wacker-type oxidation depend on specific internal alkenes bearing directing groups that favor the formation of one ketone over the other (see Fig. S1). Many of these protocols are not aerobic oxidations or depend on stoichiometric reagents such as peroxides and phenylsilanes ${ }^{10,11}$. A recently published dual-catalytic approach uses water as terminal oxidant and produces hydrogen gas as byproduct ${ }^{12}$. In general, efficient and regioselective aerobic oxidation of internal alkenes to ketones is not only a considerable challenge in catalyst development but also highly desired as it has the potential to streamline synthesis of many relevant molecules.

We have recently started to explore the potential of enzymes to directly oxidize alkenes to carbonyl compounds ${ }^{13,14}$. In a first study, we applied directed evolution to engineer an enzyme that oxidizes terminal alkenes to the corresponding aldehydes, attaining opposite selectivity to the widely used Wacker-Tsuji oxidation. The evolved iron-heme based biocatalyst achieved aldehyde formation by providing a high degree of catalyst-control in an oxo-transfer using a high-valent metal-oxo complex as catalytic oxidant (Fig. 1b). Such metaloxo complexes are utilized by many catalysts, including cytochrome P450s ${ }^{15,16}$, peroxygenases $^{17}$ and Jacobsen's catalyst ${ }^{18,19}$, to efficiently epoxidize alkenes via highly concerted reaction pathways. The key to harness oxo-transfer for carbonyl-selective alkene oxidation is to create a catalyst that is able to intercept epoxidation and couple oxo-transfer to an electron/hydride transfer process (Fig. 1b). This can be achieved by accessing a highly reactive carbocation intermediate and making it available for organic synthesis. While this is currently out of reach for small molecule catalysts, our recent study ${ }^{13}$ revealed that reactive 
centers in enzymes can be optimized with high precision to fully access and exploit this reactive intermediate. Recent mechanistic studies revealed that alkene epoxidation is a dynamically favored process and carbonyl formation can be achieved by controlling the accessible conformations of the radical and carbocation intermediates ${ }^{14}$.

a Challenge:

Regioselective oxidation of internal alkenes to ketones<smiles>[R]C=C[R]</smiles>

C Application:

Highly selective functionalization of internal alkenes

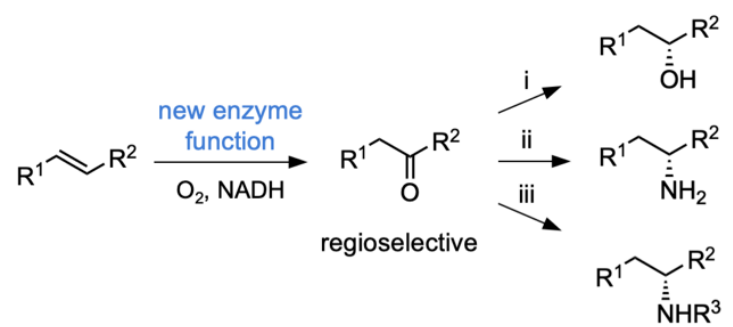

enantioselective b Catalytic strategy:

Enzyme-controlled, metal-oxo-mediated alkene oxidation
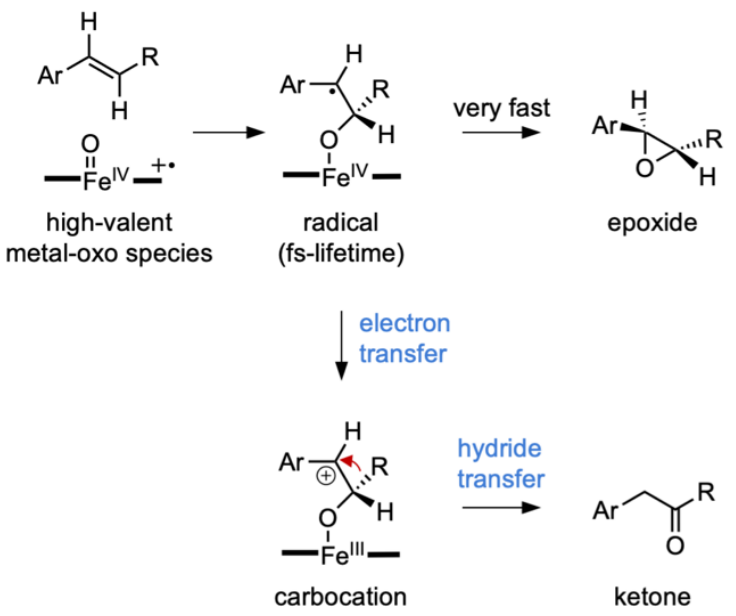

Fig. 1: Catalytic oxidation of internal alkenes to ketones. a, Regioselectivity is a challenge in catalytic oxidation of internal alkenes to ketones. b. Design of a catalytic process to convert internal alkenes to ketones using highvalent metal-oxo species as catalytic oxidant. High valent metal-oxo species typically react with alkenes in epoxidation reactions. The ketone product is accessible by stabilizing a carbocation intermediate and the reaction proceeds via an electron/hydride transfer pathway. c, Given that this new enzyme function can be accessed, many challenging functionalization reactions of internal alkenes can be realized in combination with established biocatalysts such as ketoreductases ${ }^{20,21}$ (i), $\omega$-transaminases ${ }^{20}$ (ii) and imine reductases ${ }^{22,23}$ (iii).

Here we expand this novel enzyme activity from terminal towards internal alkenes. This generates ketones with high activity and regioselectivity in a direct aerobic oxidation. Key aspects of this study are the directed enzyme evolution of a ketone synthase as well as computational studies to explain how more than a dozen beneficial mutations collaborate to access this catalytic cycle. Further, we applied the ketone synthase in synthesis as standalone catalyst to generate ketones as well as in combination with established biocatalysts in cascade reactions. The latter application enabled formal asymmetric hydrations and hydroaminations (Fig. 1c) of internal alkenes, reactions that are particularly sought after with only limited catalytic solution (Fig. S2-S3) ${ }^{24-29}$.

\section{Results and Discussion}

Directed enzyme evolution. To identify a good starting point for directed evolution, we tested various cytochrome P450 monooxygenases for activity and selectivity in the oxidation of 
internal alkenes. In particular, we focused on an inhouse mutant library of a cytochrome P450 monooxygenase from Labrenzia agreggata (P450 LA1), which has been recently evolved to oxidize terminal alkenes to aldehydes ${ }^{13}$. Trans- $\beta$-alkylstyrenes have been chosen as model substrates to expand this chemistry towards ketone synthesis. Such internal aryl alkenes are particularly challenging substrates in Wacker-type oxidation, yielding mixtures of ketone products $^{7,30}$. Indeed, we have recently confirmed initial activity with this substrate ${ }^{13}$, demonstrating that the coupled electron/hydride transfer process (Fig. 1b) can be exploited to generate ketones. The variant P7E (P450LA1-T121A-V123I-N201K-H206W-N209S-I326V$\mathrm{Y} 385 \mathrm{H}-\mathrm{E} 418 \mathrm{G}$ ) was selected from the initial screening as it showed reasonable selectivity and highest level of product formation under screening conditions (Fig. S4). Ketones are produced with a total turnover number (TTN) of 225 , yet, the performance of the catalyst is limited by the low selectivity in the reaction (Fig. 2a). In addition to phenylacetone formation (31\%), epoxidation (67\%) and allylic oxidation (2\%) were observed. It is worth mentioning that variant P7E did not produce the regioisomeric product propiophenone (Fig. S5 and computational SI material).

We aimed to optimize activity and selectivity of P7E by directed evolution. Twelve rounds of evolution uncovered 12 beneficial mutations to convert P7E into an efficient ketone synthase (Fig. 2a and 2b). The directed evolution experiment involved site-saturation mutagenesis as well as combinatorial mutagenesis libraries (Table ST1-ST2) and was performed using high-throughput UHPLC analysis. The evolved ketone synthase produced phenylacetone with high activity (2620 TTN), selectivity (75\%) and with complete regiocontrol. Allylic oxidation was a further competing reaction pathway in the course of evolution (Fig. S6 and S7), yet, the epoxide product was observed as only by-product in the final variant. Control experiments confirmed that epoxides are not isomerized by the ketone synthase (Fig. 2c and S8), supporting that catalysis proceeds via the proposed coupled electron/hydride transfer mechanism. The evolved ketone synthase is 12 -fold more active than parent P7E variant and two orders of magnitude more efficient than previously reported catalysts (Fig. S1).

The ketone synthase (KS) carries 18 mutations compared to the wild-type enzyme, 12 compared to P7E. Careful analysis revealed that 15 out of these 18 mutations are crucial to favor the ketone-forming pathway in the course of evolution (Fig. 2a and S4). Interestingly, beneficial mutations are not limited to the active site. Many mutations are in the $2^{\text {nd }}$ and $3^{\text {rd }}$ shell and their individual contributions add up to access the ketone product (Fig. $\mathbf{2 d}$ ). In addition to additive mutations, we observed cooperative effects which can be well visualized by following position 210 that changed during evolution from threonine to valine to isoleucine and back to valine, always contributing to increase selectivity and activity (Fig. 2b). ${ }^{31}$ Overall, there was no single mutation that changed the ketone-selectivity in the reaction by more than $8 \%$. 

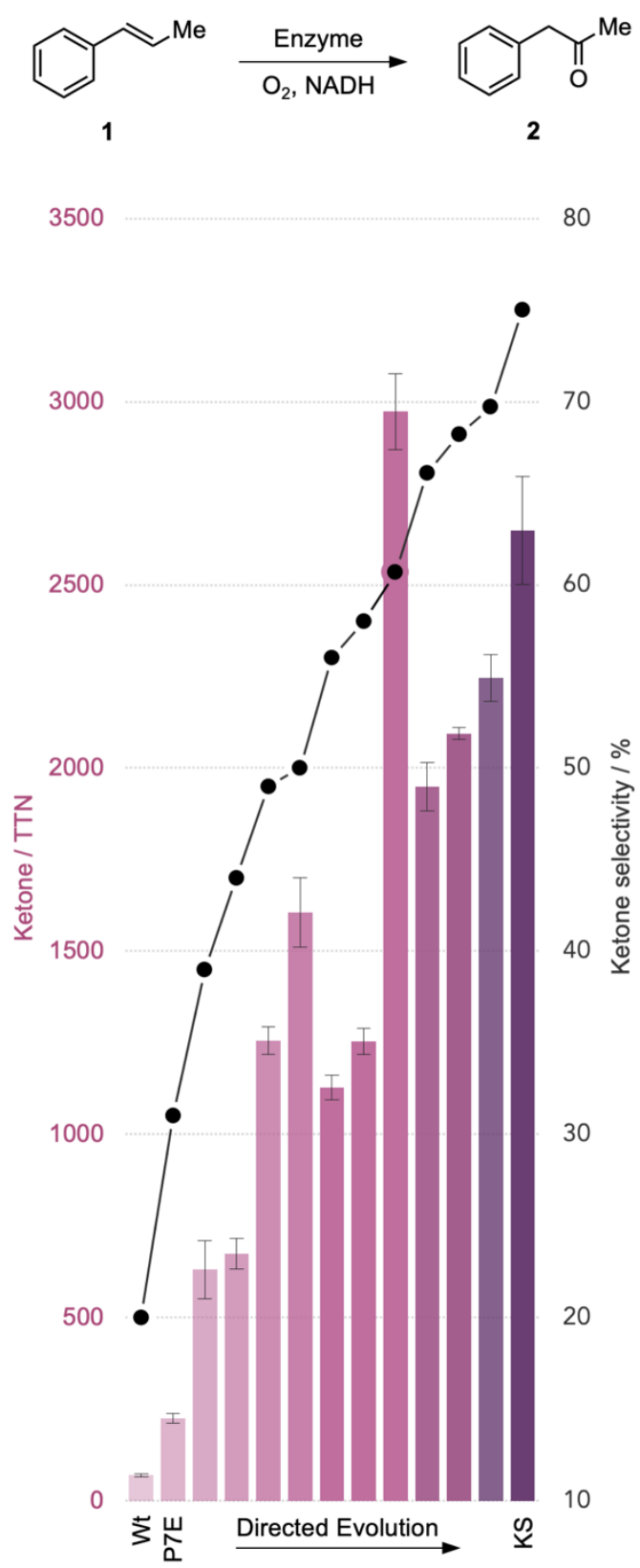

b Evolutionary Trajectory

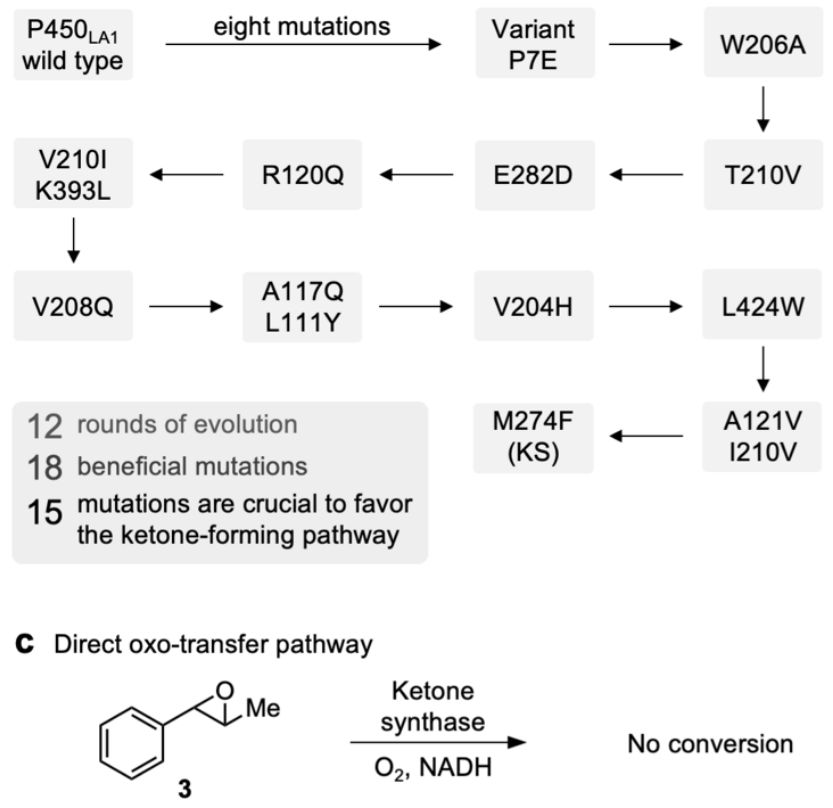

$(R, S),(S, R)$,

$(R, R)$ or $(S, S)$

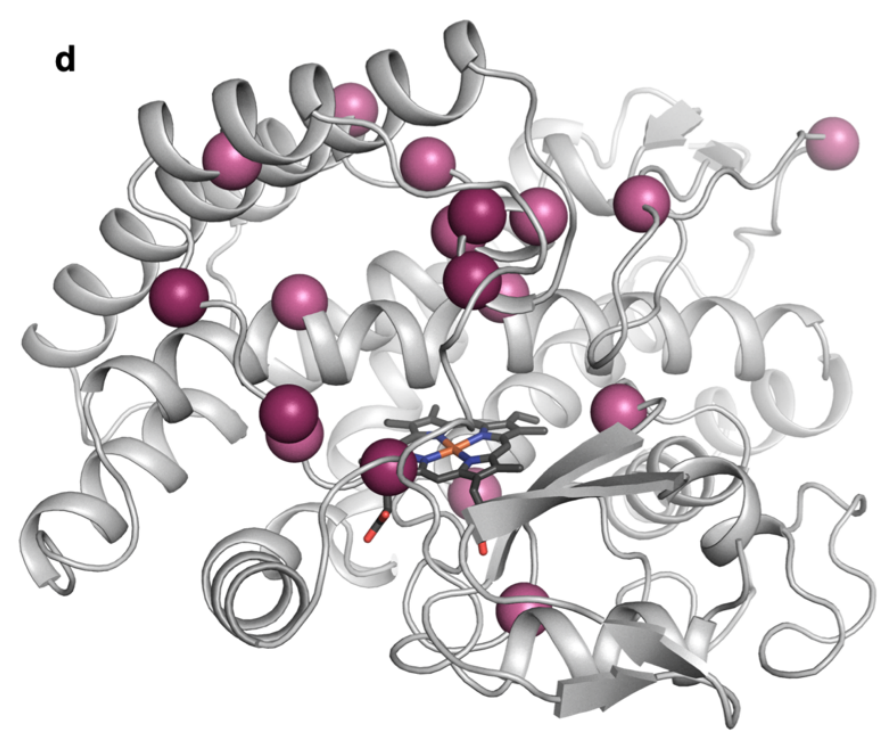

Fig. 2: Directed enzyme evolution of a ketone synthase (KS). a, Activity and selectivity as a function of evolution, starting with wild-type P450 LA1 (Wt). Biotransformations were performed with $0.625 \mu \mathrm{M}$ P450 enzyme variant, $5 \mathrm{mM}$ trans- $\beta$-methylstyrene (1), $5 \mathrm{mM} \mathrm{NADH}$ cofactor and 1 vol\% isopropanol in reaction buffer. The reactions were shaken at room temperature for $2 \mathrm{~h}$ prior to UHPLC analysis. The total turnover number (TTN) is calculated by dividing the concentration of formed ketone by the employed enzyme concentration. The ketone selectivity is determined as the proportion of ketone in relation to all oxidation products formed, including epoxides and allylic oxidation products. b. Trajectory of the directed evolution experiment listing introduced amino acid substitutions in each round. c, Epoxides are no intermediates in the reaction as they are not converted by the ketone synthase via an isomerization to the corresponding ketones. d, Homology model of P7E highlighting the introduced mutations to generate the ketone synthase as purple spheres. The heme cofactor is shown as black sticks. 
Computational modelling. In order to unravel the molecular basis of the evolved ketone synthase, we performed computational modelling. In particular, we aimed to describe the enzymatic reaction mechanism and how the multiple distal mutations contribute to fully access the carbonyl-selective reaction pathway.

We first used DFT calculations to analyze the intrinsic reaction mechanism using a computational truncated model (Fig. 3a, see computational details and complete mechanistic descriptions in the $\mathrm{SI}$ ). Calculations indicated that, as previously found for styrene and the corresponding aldehyde formation ${ }^{13,14}$, both epoxidation and ketone formation from substrate 1 share a common first step in which the first C-O bond is formed (TS1, see Fig. 3a). In the doublet electronic state, TS1 directly leads to the epoxide $\mathbf{3}$ via formation of the second C-O bond $\left(\mathrm{TS}^{\mathrm{d}}, \Delta \mathrm{G}^{\ddagger}=7.3 \mathrm{kcal} \cdot \mathrm{mol}^{-1}\right)$; in the quartet electronic state $\left(\mathrm{TS}^{9}{ }^{9}, \Delta \mathrm{G}^{\ddagger}=7.6 \mathrm{kcal} \cdot \mathrm{mol}^{-1}\right)$, a covalent radical intermediate is formed $\left(\operatorname{Int} 1^{q}, \Delta G=-13.7 \mathrm{kcal} \cdot \mathrm{mol}^{-1}\right)$. This radical intermediate Int ${ }^{q}$ (Fig. 3a) can follow the epoxidation pathway forming the second $\mathrm{C}-\mathrm{O}$ bond through a low in energy TS $\left(\mathrm{TS2}^{q}, \Delta \mathrm{G}^{\ddagger}=1.6 \mathrm{kcal} \cdot \mathrm{mol}^{-1}\right)$, or form a covalent carbocation intermediate via an intramolecular electron transfer from the substrate moiety to the $\mathrm{Fe}$ porphyrin (Int2 ${ }^{\mathrm{q}}$ and $\operatorname{Int} \mathbf{2}^{\mathrm{d}}, \Delta \mathrm{G}=3.0$ and $-2.2 \mathrm{kcal} \cdot \mathrm{mol}^{-1}$, respectively). In this truncated model, the formation of carbocation Int2 is triggered by a conformational change, namely the rotation of the phenyl ring of the substrate. This allows optimal stabilization of the benzylic carbocation by maximizing hyperconjugation with the neighboring $\sigma(\mathrm{C}-\mathrm{H})$ and $\sigma(\mathrm{C}-\mathrm{C})$ bonds as well as resonance with the aromatic ring, and by $n \rightarrow p$ interactions between the oxygen lone pairs and the empty carbocation $p$ orbital (Fig. $\mathbf{3 b}$ and Fig. S17) $)^{14}$. In this geometry, an intramolecular $\mathrm{H}$-bond between the ortho-hydrogen of the phenyl ring and the oxygen atom is also established. Once formed, the carbocation within this conformation can undergo a barrierless 1,2-hydride migration to form the final ketone product 2 . DFT calculations indicated that $\mathrm{i}$ ) the epoxidation pathway is slightly energetically favored over ketone formation, although both pathways being very similar in energy and ii) catalytic access to the ketone product depends on stabilizing the reactive intermediates in a specific conformation.

Previous computational modelling proposed that the strong preference for epoxide formation is a consequence of the dynamic behavior of the radical intermediate, which is formed with an excess of energy that is not statistically distributed before further reacting ${ }^{14}$. The covalent radical intermediate formed from TS1 is kinetically activated, possessing a momentum vector that matches the one for the epoxide forming transition state (TS2), as previously shown ${ }^{14}$. This strong "dynamic match" favors alkene epoxidation over carbonyl formation that requires a specific carbocation geometry to promote the 1,2-hydride migration (Fig. 3b). Consequently, we hypothesized that carbonyl-selectivity achieved by the evolved ketone synthase derives from conformational control over the intermediates due to geometric restrictions in the enzyme active site ${ }^{14}$. 


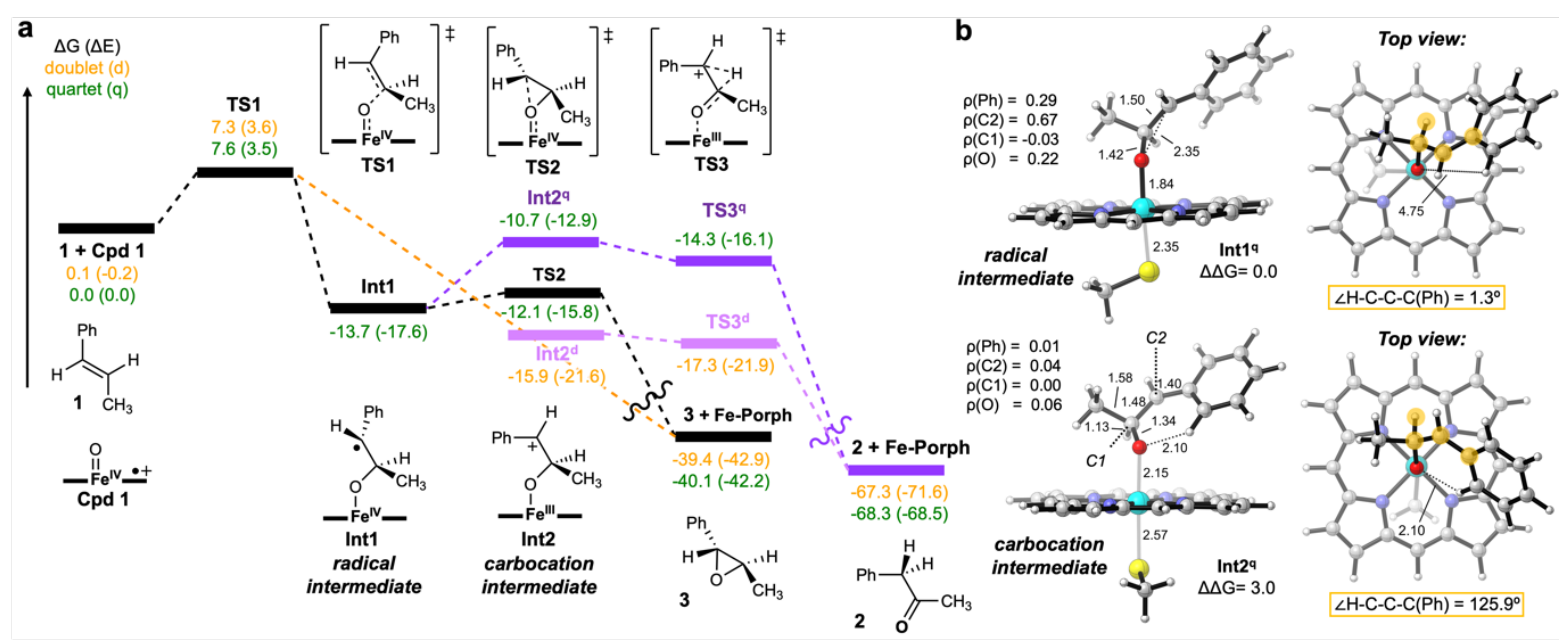

Figure 3. DFT calculations of the intrinsic reaction mechanism for ketone formation using a computational truncated model. A, Calculated energy profile (free Gibbs energy, $\Delta \mathrm{G}$, and electronic energy, $\Delta \mathrm{E}$ ) for the competing epoxidation and ketone formation pathways for trans- $\beta$-methylstyrene (1). Energies for doublet and quartet electronic states are reported in $\mathrm{kcal} \cdot \mathrm{mol}^{-1}$. B, DFT-optimized structures for the radical (Int1) and carbocation (Int2) intermediates and their relative free energies $(\Delta \Delta \mathrm{G})$. Key distances, dihedral angle (highlighted in yellow), and spin densities $(\rho)$ are given in angstroms $(\AA)$, degrees $\left(^{\circ}\right)$, and atomic units (a.u.), respectively.

In order to study the enzymatic reaction, homology models for P7E and KS were first constructed (see details in the SI). Extensive MD simulations (5 independent replicas of 1,000 ns each, 5,000 ns in total for each system) revealed important changes in the overall structure between KS and parent enzyme P7E (see Fig. S19), which also affected the shape of the active site cavity. Visual inspection of the active site in the evolved KS describes a more confined substrate binding pocket compared to parent P7E (Fig. 4a and S20). This reshaped active site is expected to improve binding of substrate 1 in a reactive conformation, and might also be important to control the conformations of the subsequently formed reactive intermediates.

Next, we aimed to characterize the reactive binding conformations of the substrate in P7E and KS active sites. We used substrate 1 in docking calculations, which were refined by extensive restrained-MD simulations (5 replicas of $500 \mathrm{~ns}, 2,500 \mathrm{~ns}$ in total) mimicking near attack conformations (NAC) leading to the key TS1 (see SI for details). Simulations revealed that the ketone synthase shows improved substrate binding in this reactive conformation achieved by a more packed active site compared to P7E (Fig. 4b and Figs. S21 and S22). This increased packing is reflected by two different observations. First, a more pronounced rigidification was observed for the KS active site residues upon substrate binding in the NAC conformation (Fig. 4c, $\triangle$ RMSF). This is especially highlighted by 121-124 residues of the B'Cloop region and the 273-284 residues in the I-helix (Figs. 4c, and Fig. S23). Second, the strengthened interactions occurring in KS active site between the substrate and surrounding residues (Fig. 4c and S24). The substrate bound in the NAC conformation shows stronger 
interactions with active site residues L97, A121V, W211, A275, or I276 in the active site of KS compared to P7E. All these residues are placed around the phenyl ring of the substrate, and consequently restrain its conformation (Fig. 4b). Overall, the observed increased packing of the KS active site favors substrate binding in the appropriate reactive near attack conformation.

a MD simulations

b Restrained-MD simulations
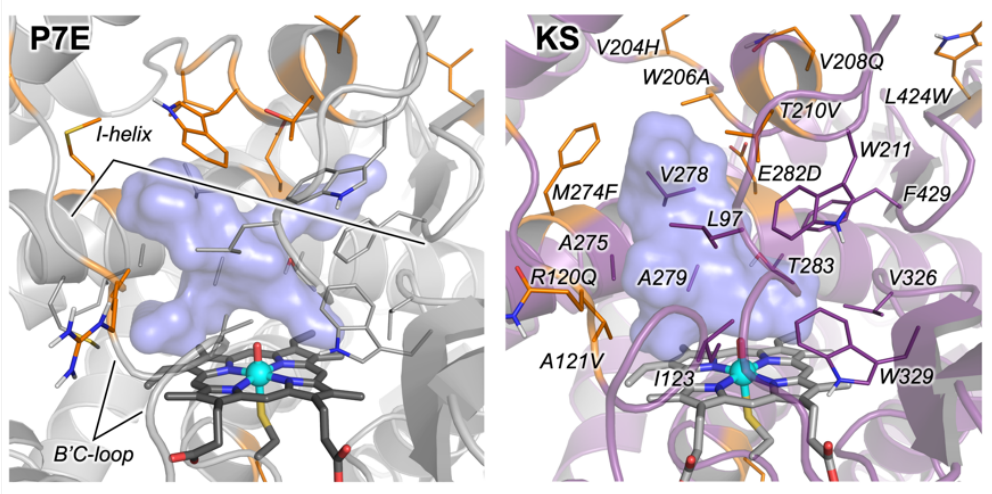

ks

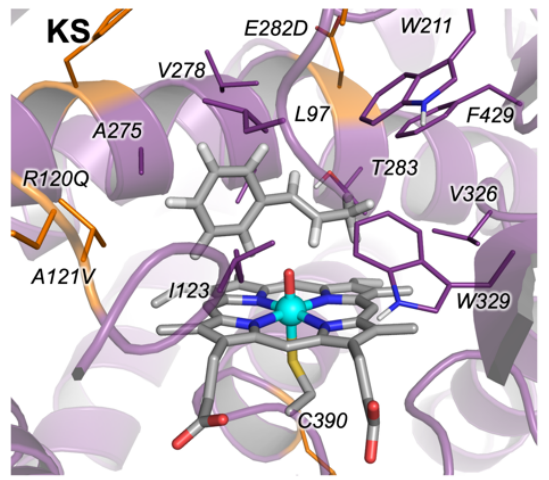

C

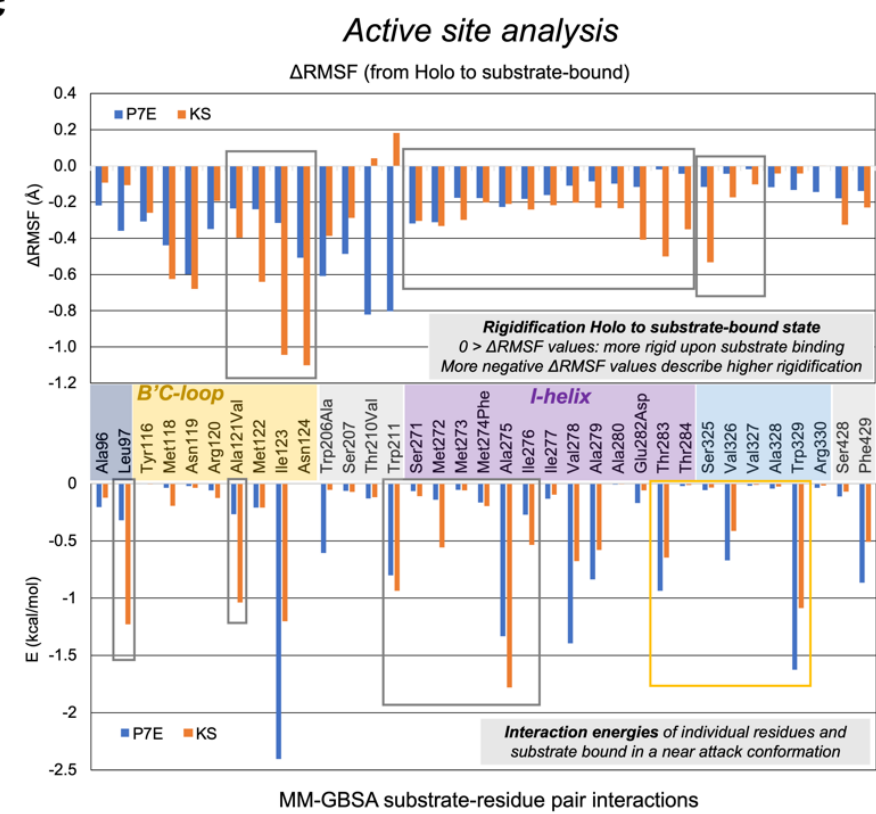

d

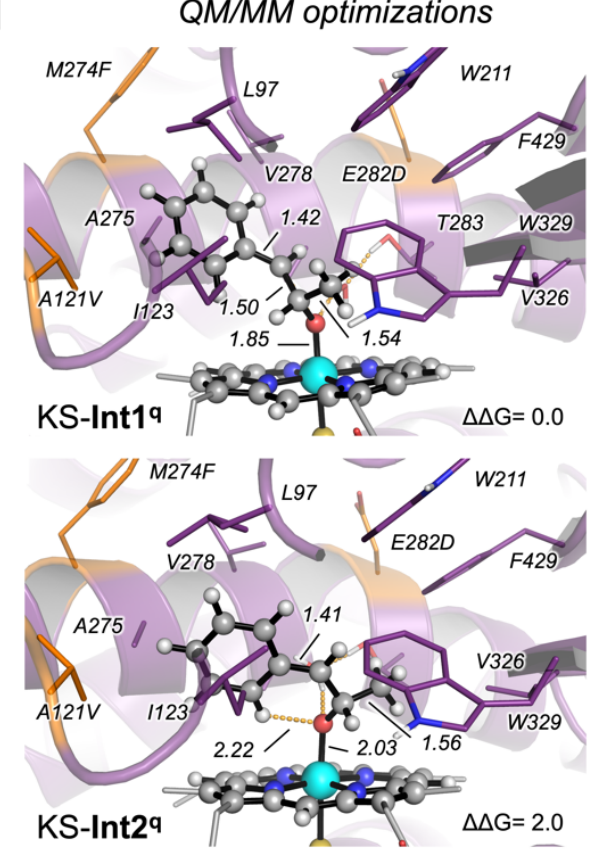

Figure 4. Analysis of the active site of the evolved ketone synthase. a, Active site arrangement in P7E and KS variants characterized from MD simulations in their holo states. Cavity surface is shown in purple. b, Representative structure obtained from restrained-MD simulations for trans- $\beta$-methylstyrene (1) bound in a reactive near attack conformation (NAC) in the KS active site. c, Analysis of active site residues from accumulated MD trajectories. $\triangle$ RMSF describe the root mean square fluctuation (RMSF) measured for active site residues along NAC substrate-bound MD simulations, as compared to holo state simulations (RMSF(substrate-bound) $R M S F(h o l o)$ ). The more negative the $\triangle R M S F$, the more rigid (i.e. less mobile) the residue becomes after binding the substrate in the simulated near attack conformation. MM-GBSA substrate-residue pair interaction energies describe the strength of the interactions between trans- $\beta$-methylstyrene and surrounding active site residues along the NAC substrate-bound MD simulations. d, QM/MM optimized geometries for the radical intermediate (KS-Int19) and carbocation intermediate (KS-Int2 ${ }^{\mathrm{q}}$ ) formed in KS active site. Relative Gibbs free energies $(\Delta \Delta G)$ and key distances are given in $\mathrm{kcal} \cdot \mathrm{mol}^{-1}$ and angstroms $(\AA)$, respectively. 
The enhanced active site packing plays also a crucial role in controlling the radical intermediate formed from the common TS1. First, tight interactions with surrounding residues help the intermediate to dissipate its excess of vibrational energy and achieve thermal equilibration once formed ${ }^{14}$. Second, active site tightening help to geometrically preorganize the radical intermediate to access the required conformation for the effective formation of the carbocation intermediate while disfavoring the formation of the second C-O bond. These findings are supported by QM/MM calculations carried out on the evolved ketone synthase (see the complete QM/MM mechanism in the SI, Fig. S28). In summary, we found that geometric restrictions imposed by the packed KS active site favor the carbocation intermediate while destabilizing the epoxide forming transition state (see Fig. S28) as compared to the freeenzyme model (Fig. 3a). The QM/MM optimized radical intermediate formed in the KS active site $\left(\mathrm{KS}-\operatorname{Int} 1^{\mathrm{q}}\right)$ has the phenyl group tightly packed between the same amino acids of the B'Cloop region and I-helix as identified by NAC analysis of the substrate (see Fig. 4d). These steric interactions between the radical intermediate and the active site facilitate access to the geometry required for effective carbocation formation (KS-Int2q, Fig. 4d), and increase the barrier for the second C-O bond formation (Fig. S28). This geometry allows stabilization of the carbocation intermediate due to stereoelectronic effects and aligns the cis- $\beta$-hydrogen atom with the empty carbon $p$-orbital for a fast and selective 1,2-hydride migration (see Fig. S28). In addition, computations show that the presence of an ordered water molecule in the active site can promote the carbocation formation even more (see Fig. S29).

In order to rationalize how numerous distal mutations (Fig. 2d) optimized the active site, we have studied the enzyme conformational dynamics using dynamical correlation networks in P7E and KS variants. Dynamical networks describe connections between distal protein residues based on their correlated dynamic behavior mediated by neighboring residues (in sequence or 3D-space). It has been proposed that dynamical networks can modulate the transition between active and inactive conformations of enzymes ${ }^{32}$, and impact the active site preorganization by controlling enzyme conformational flexibility and reducing non-productive conformations $^{33}$.

Analysis of the holo enzymes revealed the emergence of an expanded dynamical network in KS as compared to P7E. Shortest path maps (SPM) ${ }^{34}$, which describe communications between neighboring residues, show that the new enlarged dynamical network in $\mathrm{KS}$ is centered on the substrate binding pocket and completely surrounds it (Fig. 5 and S25). The expanded dynamical network includes residues placed on the I-helix and the 116-124 B'Cloop region that were not part of the original network but are important for the conformational control of the substrate and the intermediates (Fig. 4c). The enhancement and tuning of the protein dynamical network can be attributed to the newly introduced mutations, which is in line with previous studies, ${ }^{32-35}$ including important epistatic effects as in related P450s that directly 
impact on catalysis. ${ }^{31}$ Although only four mutations appear as nodes of the SPM in KS, practically all of the introduced mutations, including the distal ones, appear at adjacent positions in 3D space (Fig. S25 and S26), thus contributing to the proteins dynamic behavior ${ }^{32-}$ 35. The enhanced dynamical network in KS is not only maintained but also strengthened in the reactive substrate-bound state (Fig. 5 and S26), similarly to what has been observed in other laboratory evolved enzymes ${ }^{33,36}$. Taking all this into account, the observed reshaping and rigidification of the active site in $\mathrm{KS}$ can be attributed to the changes in the dynamical network of the evolved enzyme.

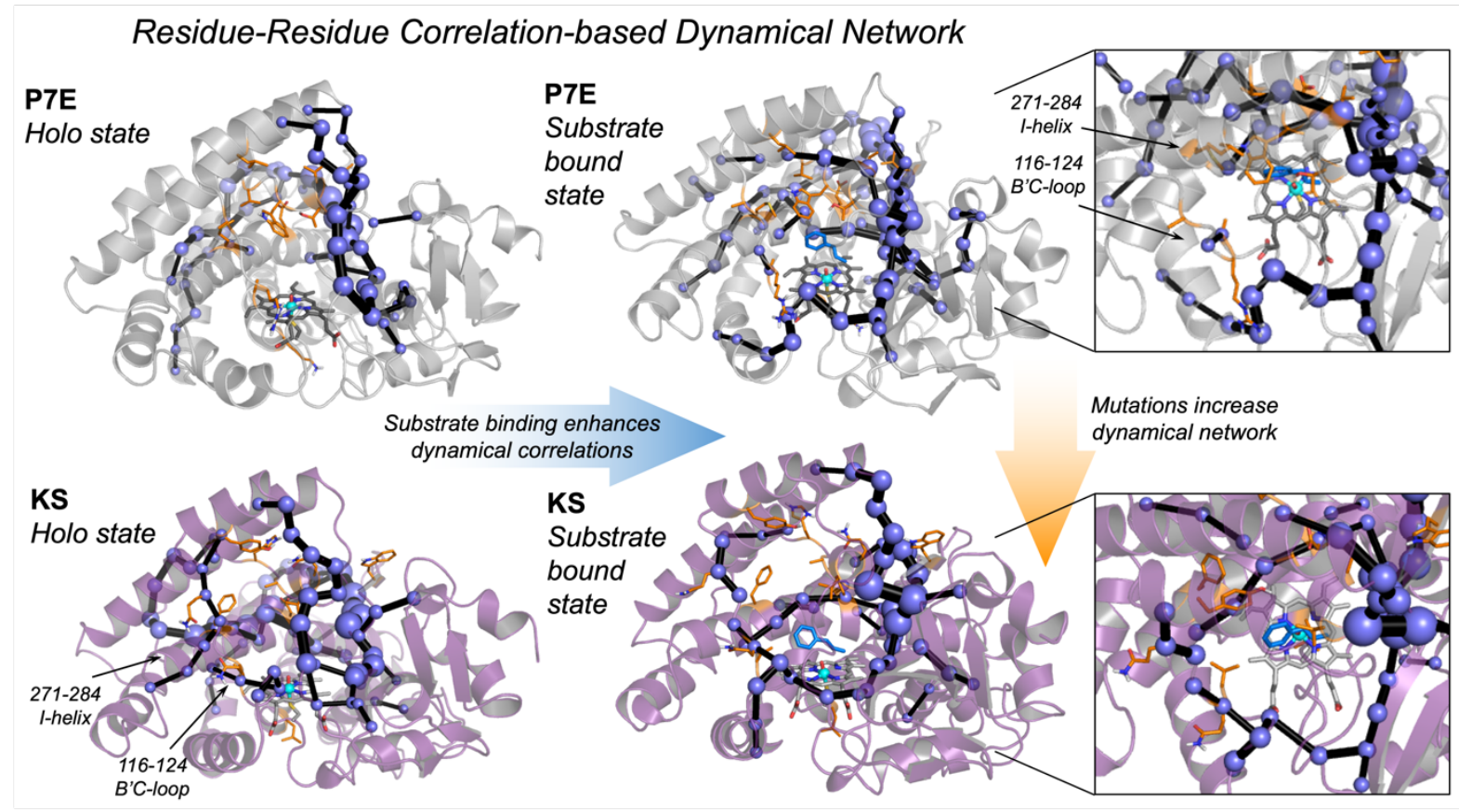

Figure 5. Correlation-based dynamical network analysis through shortest path maps (SPM). Shortest path maps $^{34}$ calculated from accumulated MD simulation time for P7E and KS variants in their holo and "substratebound in a near attack conformation" states. The sizes of the spheres (nodes of the network) and black lines (edges of the network) are indicative of their weight in the network. Positions mutated during evolution are shown in stick format and highlighted in orange. Heme cofactor and substrate $\mathbf{1}$ are shown in sticks format, gray and cyan, respectively.

Substrate scope and application in synthesis. To understand the application and limitation of the ketone synthase in synthesis, we investigated its substrate scope with various substituted $\beta$-alkyl styrenes (Fig 6a). The tested substrates were converted by the ketone synthase with efficiencies ranging up to several thousand TTNs and selectivities up to $79 \%$. Various substitutions are tolerated including substitutions in ortho- (11), meta-(10) and paraposition (4-8) of the aromatic ring as well as in trans- $\beta$-position (9). Cis- $\beta$-methylstyrene was converted by the ketone synthase yielding epoxides but not phenylacetone (Fig. S9). This is in agreement with recent mechanistic findings highlighting that the hydride migrates selectively from the cis-position ${ }^{14}$. Further, substrates bearing substitutions in $\alpha$-position enabled 
synthesis of the chiral ketone 12 with good enantioselectivity, albeit with low activity (Fig. S10). Stereoselectivity is achieved by enantiofacial discrimination in the 1,2-hydride migration, in line with previous observations ${ }^{14}$. The selectivity detected is also consistent with the selectivity model proposed based on the computational analysis carried out for trans- $\beta$-methylstyrene (Fig. S11). It is worth highlighting that catalytic asymmetric oxidation of internal alkenes to chiral ketones are currently unknown.

The activity of the ketone synthase could be further optimized by changing reaction conditions. Elongation of the reaction time and application of a cofactor recycling system enabled conversion of 1 to phenylacetone with up to 4750 TTN (Fig. S12). To demonstrate that these reactions can be performed on a preparative scale $(1.0 \mathrm{mmol})$, ketone 2 was synthesized using a catalyst loading of $0.025 \mathrm{~mol} \%$ ketone synthase (Fig. 6b). The product was isolated with $61 \%$ yield, consuming atmospheric oxygen and D-glucose as only stoichiometric reagents.

a Substrate Scope<smiles>CC=Cc1ccccc1</smiles>

1<smiles>CC(=O)Cc1ccc(Br)cc1</smiles>

4 1840 TTN, $79 \%$
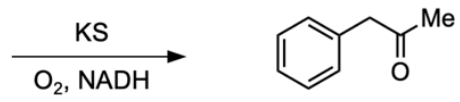
2620 TTN $^{2}$ 75\%
4750 TTN $^{*}$

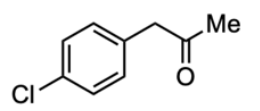

5 1670 TTN, $79 \%$<smiles>CC(=O)Cc1ccc(F)cc1</smiles>

6 1000 TTN, $72 \%$<smiles>CC(=O)Cc1ccc(C(F)(F)F)cc1</smiles>

350 TTN, $48 \%$<smiles>COc1ccc(CC(C)=O)cc1</smiles>
360 TTN, $12 \%$<smiles>CCC(=O)Cc1ccccc1</smiles>
500 TTN, $47 \%$<smiles>CC(=O)Cc1cccc(Cl)c1</smiles>

10

25 TTN, $25 \%$<smiles>CC(=O)Cc1ccccc1Cl</smiles>

11 640 TTN, $71 \%$<smiles>CC(=O)C(C)c1ccccc1</smiles>

12

10 TTN, 3\% $87: 13$ (S:R) e.r. b Preparative Scale Reactions (1.0 mmol scale, 0.025 mol\% ketone synthase)<smiles>CC(=O)Cc1ccccc1</smiles>

2 Yield: $61 \%$ $68 \%$ product form<smiles>CC=Cc1ccccc1</smiles>

1

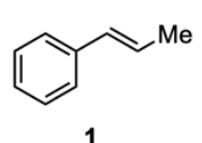

1
$\underset{\mathrm{NAD}, \mathrm{PAR}}{\stackrel{\mathrm{K}}{\mathrm{O}} \mathrm{O}_{2}}$ $i-\mathrm{PrOH}$

$\mathrm{KS}$, $\underset{\mathrm{NAD}+}{\stackrel{\mathrm{LBv}}{\mathrm{O}} \mathrm{O}_{2},}$ i-PrOH Yield: $69 \%$ $74 \%$ product form $>99: 1(R)$ e.r.

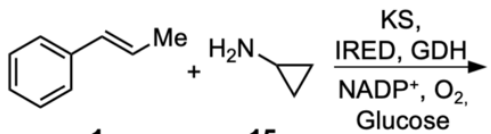

1<smiles>C[C@H](Cc1ccccc1)NC1CC1</smiles>

16 Yield: $39 \%$ $41 \%$ product form. 92:8 (S:R) e.r.

Fig. 6: Application in synthesis. a, Substrate scope of the ketone synthase (KS). Reactions were carried out using $0.625 \mu \mathrm{M} \mathrm{KS}, 5 \mathrm{mM}$ of the corresponding substrate and $5 \mathrm{mM}$ NADH cofactor. Total turnover numbers (TTN) were determined after $2 \mathrm{~h}$ reaction time. *Maximum TTN was determined after $48 \mathrm{~h}$ biotransformation combined with a cofactor regeneration system. $\mathbf{b}$, Application on preparative scale to synthesize ketones as well as in formal asymmetric hydration and hydroamination of internal alkenes. Please see SI (Fig. S14-S15) for more details. GDH: Glucose dehydrogenase; PAR: Phenylacetaldehyde reductase; $L B V-A D H$ : Alcohol dehydrogenase from Lactobacillus brevis, IRED: Imine reductase pIR-23 (CfIRED). 
Formal asymmetric hydration and hydroamination of internal alkenes. Laboratory evolution of non-natural enzyme function can provide access to new synthetic pathways and solve long-standing challenges in synthesis ${ }^{37-39}$. In this regard, we performed asymmetric redox hydrations and hydroaminations of internal alkenes by combining the ketone synthase with ketoreductase $\mathrm{s}^{20,21}$ and imine reductase $\mathrm{s}^{22,23}$ on preparative scale $(1.0 \mathrm{mmol})$. With this setup, the unactivated internal alkene 1 was converted to chiral phenylethanols and phenylethylamine that are important structural motifs in top-selling pharmaceuticals (Fig. S13). The chiral alcohols 13 and 14 as well as amine 16 were produced with excellent enantioselectivity (up to $>99: 1$ e.r.) and with 66,69 and $39 \%$ isolated yield, respectively (Fig. 6b). An important feature of these reactions is that i) they only depend on simple stoichiometric reagents such as atmospheric oxygen and isopropanol (asymmetric hydration) or atmospheric oxygen and glucose (asymmetric hydroamination) and ii) simple unprotected amines can be used as amine donors. This approach can in principle be expanded to other internal alkenes (Fig. 6a), or other amines as amine donors ${ }^{23,40}$.

In addition, we aimed to generate two stereocenters using the trisubstituted trans-alkene 17 as starting material (Fig. 7). Alcohol 18 was obtained via ketone 12 with low activity but excellent selectivity yielding one single stereoisomer. This approach combines the KScatalyzed enantioselective alkene to ketone oxidation via an asymmetric hydride migration with the diastereofacial differentiation of the ketoreductase.

Such conversions of unactivated internal alkenes to chiral alcohols and amines are a particular challenge in catalysis (Fig. S2-S3) with only limited catalytic solutions ${ }^{24-29}$. There are natural enzymes that catalyze direct hydroaminations of internal alkenes, yet, due to their underlying mechanism they depend on activated internal alkenes such as $\alpha, \beta$ unsaturated carboxylic acids ${ }^{41,42}$. As internal unactivated alkenes are easily accessible and ketones are substrates in many chemical transformations using enzymes ${ }^{21,23,43-46}$ or small molecule catalysts $^{47,48}$, many new synthetic routes can be envisioned using evolved ketone synthases. 
<smiles>[M]C=C(C=[W])c1ccccc1</smiles>

17

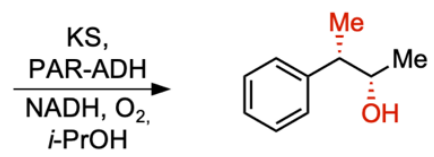

18

$1.8 \mu \mathrm{M}$ (low conversion) single stereoisomer

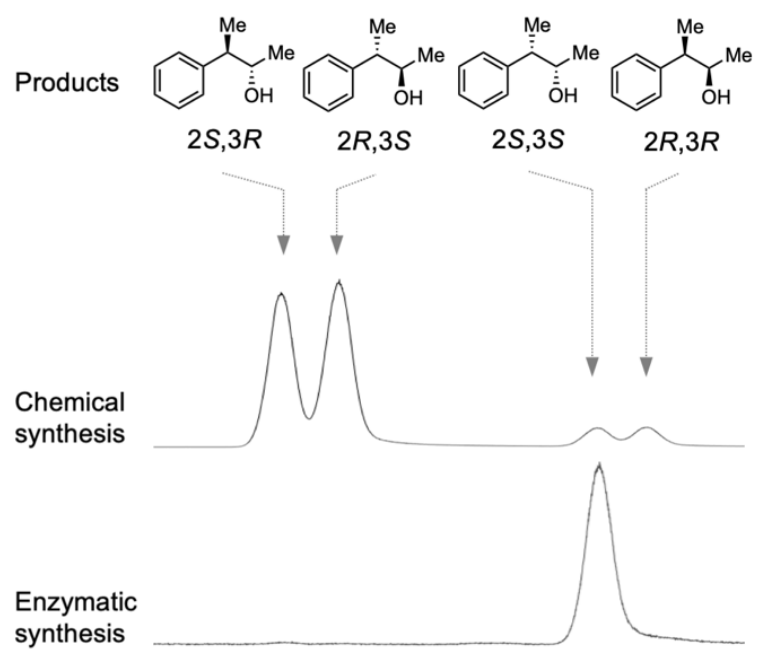

Fig. 7: Formal asymmetric hydration of trisubstituted trans-alkene 17 to generate two stereocenters. The enzymatic reaction generates a single stereoisomer of alcohol 18 via ketone 12 and combines the enantioselective oxidation of the ketone synthase (KS) with the diastereofacial discrimination of a ketoreductase (phenylacetaldehyde reductase, PAR). The enzymatic reaction is compared to a chemical synthesis that generates all possible stereoisomers (see Fig. $\mathbf{S 1 6}$ for more information).

\section{Conclusions}

Our findings demonstrate that cytochrome P450 can be evolved to oxidize internal alkenes to ketones with high activity and selectivity. We applied the evolved ketone synthase to directly convert internal alkenes to ketones, including a first catalytic enantioselective example. In addition, we combined the ketone synthase with established biocatalysts to convert internal alkenes to chiral phenylethanols and phenylethylamines on preparative scale with high activity, regio- and enantioselectivity. What stands out is that the ketone synthase enables many important, highly selective functionalization reactions of internal alkenes which have so far largely eluded efficient and selective catalysis.

Directed evolution of protein-based catalysts can exert an exceptional degree of control over reactive intermediates. This is achieved in the ketone synthase evolution by generating a confined, rigid and preorganized active site through multiple mutations that enhance a preexisting dynamical network. The evolved ketone synthase provides access to a highly reactive carbocation species and thus enables new chemistry for ketone production. We foresee that the confinement ${ }^{49}$ of protein-based catalysts can be generally leveraged to enable other challenging catalytic cycles by precisely controlling the accessible conformations of reactive 
intermediates. One of the next exciting question for us is whether the coupled oxo/electron/hydride transfer process can also be fully utilized with less reactive alkylsubstituted alkenes as substrates.

\section{Acknowledgements}

We thank Matúš Gajdoš, Cindy Klaus, Dr. Bettina Nestl and Felipe Ospina for fruitful discussion and comments to improve the manuscript. We further acknowledge Michael Breuer (BASF SE) for disclosing sequence information for a ketoreductase as well as Harald Gröger (Bielefeld University) and Nicholas Turner (University of Manchester) for providing plasmid DNA coding for glucose dehydrogenases and imine reductases, respectively. This work was supported by the Deutsche Forschungsgemeinschaft via the Emmy Noether program (fellowship HA 7668/2-1), the Generalitat de Catalunya AGAUR for a Beatriu de Pinós H2020 MSCA-Cofund 2018-BP-00204 project (to M.G.B.), and the Spanish MICINN (Ministerio de Ciencia e Innovación) for PID2019-111300GA-I00 project (to M.G.B.). J. S. thanks the Spanish MIU (Ministerio de Universidades) for a predoctoral FPU fellowship FPU18/02380. The computer resources at the Barcelona Supercomputing Center BSC-RES are acknowledged (RES-QSB-2019-3-0009, RES-QSB-2020-2-0016, and RES-QH-2021-2-0013).

\section{Author contributions}

S.C.H. led the project. S.G. and S.C.H. designed the wet-lab experiments. S.G. performed experiments. S.G. and S.C.H. analyzed data. M.G.B. designed and supervised the computational modelling. J.S. and M.G.B. performed the computational work and analyzed the data. A.K. and K.H.S. have contributed to compound synthesis and substrate scope studies. S.G., S.C.H., M.G.B. and J.S. co-wrote the manuscript and all authors agreed on the final version.

\section{Literature}

1. Smith, M. B. \& March, J. March's advanced organic chemistry. (Wiley-VCH, 2007).

2. Grubbs, R. H. \& Wenzel, A. G. Handbook of Metathesis: Second Edition. (Wiley-VCH, 2015).

3. Tekada, T. Modern Carbonyl Olefination: Methods and Applications. (Wiley-VCH, 2006).

4. Atkins, W. J., Burkhardt, E. R. \& Matos, K. Safe handling of boranes at scale. Org. Process Res. Dev. 10, 1292-1295 (2006).

5. Burkhardt, E. R. \& Matos, K. Boron reagents in process chemistry: Excellent tools for selective reductions. Chem. Rev. 106, 2617-2650 (2006).

6. Morandi, B., Wickens, Z. K. \& Grubbs, R. H. Regioselective Wacker oxidation of internal alkenes: rapid access to functionalized ketones facilitated by cross-metathesis. Angew. Chem. Int. Ed. 52, 9751-9754 (2013).

7. Morandi, B., Wickens, Z. K. \& Grubbs, R. H. Practical and general palladium-catalyzed synthesis of 
ketones from internal olefins. Angew. Chem. Int. Ed. 52, 2944-2948 (2013).

8. Deluca, R. J. et al. Wacker-type oxidation of internal alkenes using Pd(Quinox) and TBHP. J. Org. Chem. 78, 1682-1686 (2013).

9. Mitsudome, T., Mizumoto, K., Mizugaki, T., Jitsukawa, K. \& Kaneda, K. Wacker-type oxidation of internal olefins using a $\mathrm{PdCl} / \mathrm{N}, \mathrm{N}$-dimethylacetamide catalyst system under copper-free reaction conditions. Angew. Chem. Int. Ed. 49, 1238-1240 (2010).

10. Puls, F. \& Knölker, H. Conversion of Olefins into Ketones by an Iron-Catalyzed Wacker-type Oxidation Using Oxygen as the Sole Oxidant. Angew. Chem. Int. Ed. 57, 1222-1226 (2018).

11. Puls, F., Linke, P., Kataeva, O. \& Knölker, H. Iron-Catalyzed Wacker-type Oxidation of Olefins at Room Temperature with 1,3-Diketones or Neocuproine as Ligands**. Angew. Chem. Int. Ed. 60, 14083-14090 (2021).

12. Zhang, G. et al. Anti-Markovnikov oxidation of $\beta$-alkyl styrenes with $\mathrm{H} 2 \mathrm{O}$ as the terminal oxidant. J. Am. Chem. Soc. 138, 12037-12040 (2016).

13. Hammer, S. C. et al. Anti-Markovnikov alkene oxidation by metal-oxo-mediated enzyme catalysis. Science 358, 215-218 (2017).

14. Soler, J., Gergel, S., Hammer, S. C. \& Garcia-Borràs, M. Mechanistic insights into anti-Markovnikov alkene oxidations by engineered P450s: enzymatic control of reactive intermediates. Manuscript in preparation (2022).

15. Guengerich, F. P. Mechanisms of cytochrome P450-catalyzed oxidations. ACS Catal. 8, 10964-10976 (2018).

16. Ortiz De Montellano, P. R. Cytochrome P450: Structure, mechanism, and biochemistry. Cytochrome P450: Structure, mechanism, and biochemistry (Springer US, 2005).

17. Wang, Y., Lan, D., Durrani, R. \& Hollmann, F. Peroxygenases en route to becoming dream catalysts. What are the opportunities and challenges? Curr. Opin. Chem. Biol. 37, 1-9 (2017).

18. Zhang, W., Loebach, J. L., Wilson, S. R. \& Jacobsen, E. N. Enantioselective epoxidation of olefins catalyzed by (salen)manganese complexes. J. Am. Chem. Soc. 112, 2801-2803 (1990).

19. Jacobsen, E. N., Zhang, W., Muci, A. R., Ecker, J. R. \& Deng, L. Highly enantioselective epoxidation catalysts derived from 1,2-diaminocyclohexane. J. Am. Chem. Soc. 113, 7063-7064 (1991).

20. Nestl, B. M., Hammer, S. C., Nebel, B. A. \& Hauer, B. New generation of biocatalysts for organic synthesis. Angew. Chem. Int. Ed. 53, 3070-3095 (2014).

21. Wu, S., Snajdrova, R., Moore, J. C., Baldenius, K. \& Bornscheuer, U. T. Biocatalysis: enzymatic synthesis for industrial applications. Angew. Chem. Int. Ed. 60, 88-119 (2021).

22. Sharma, M., Mangas-Sánchez, J., Turner, N. J. \& Grogan, G. NAD(P)H-dependent dehydrogenases for the asymmetric reductive amination of ketones: structure, mechanism, evolution and application. Adv. Synth. Catal. 359, 2011-2025 (2017).

23. Ducrot, L., Bennett, M., Grogan, G. \& Vergne-Vaxelaire, C. NAD(P)H-dependent enzymes for reductive amination: active site description and carbonyl-containing compound spectrum. Adv. Synth. Catal. 363, 328-351 (2021).

24. Hintermann, L. Recent developments in metal-catalyzed additions of oxygen nucleophiles to alkenes and alkynes. Top. Organomet. Chem. 31, 123-155 (2010).

25. Demming, R. M. et al. Asymmetric enzymatic hydration of unactivated, aliphatic alkenes. Angew. Chem. Int. Ed. 58, 173-177 (2019).

26. Musacchio, A. J. et al. Catalytic intermolecular hydroaminations of unactivated olefins with secondary alkyl amines. Science 730, 727-730 (2017).

27. Pirnot, M. T., Wang, Y.-M. \& Buchwald, S. L. Copper hydride-catalyzed hydroamination of alkenes and alkynes. Angew. Chem. Int. Ed. 55, 48-57 (2015).

28. Xi, Y., Ma, S. \& Hartwig, J. F. Catalytic asymmetric addition of an amine $\mathrm{N}-\mathrm{H}$ bond across internal alkenes. Nature 588, 37-40 (2020).

29. Yang, Y., Shi, S.-L., Niu, D., Liu, P. \& Buchwald, S. L. Catalytic asymmetric hydroamination of unactivated internal olefins to aliphatic amines. Science 349, 62-66 (2015).

30. Keinan, E., Seth, K. K. \& Lamed, R. Organic synthesis with enzymes. 3. TBADH-catalyzed reduction of chloro ketones. Total synthesis of (+)-(S,S)-(cis-6-methyltetrahydropyran-2-yl)acetic acid: a civet constituent. J. Am. Chem. Soc. 108, 3474-3480 (1986).

31. Acevedo-Rocha, C. G. et al. Pervasive cooperative mutational effects on multiple catalytic enzyme traits emerge via long-range conformational dynamics. Nat. Commun. 12, 1621 (2021).

32. Osuna, S. The challenge of predicting distal active site mutations in computational enzyme design. Wiley Interdiscip. Rev. Comput. Mol. Sci. 11, 1-20 (2021). 
33. Bunzel, H. A. et al. Evolution of dynamical networks enhances catalysis in a designer enzyme. Nat. Chem. 13, 1017-1022 (2021).

34. Romero-Rivera, A., Garcia-Borràs, M. \& Osuna, S. Role of Conformational Dynamics in the Evolution of Retro-Aldolase Activity. ACS Catal. 7, 8524-8532 (2017).

35. Maria-Solano, M. A., Iglesias-Fernández, J. \& Osuna, S. Deciphering the Allosterically Driven Conformational Ensemble in Tryptophan Synthase Evolution. J. Am. Chem. Soc. 141, 13049-13056 (2019).

36. Calvó-Tusell, C., Maria-Solano, M. A., Osuna, S. \& Feixas, F. Unravelling the Graded Millisecond Allosteric Activation Mechanism of Imidazole Glycerol Phosphate Synthase. bioRxiv (2021) doi:10.1101/2021.10.21.464958.

37. Erb, T. J., Jones, P. R. \& Bar-Even, A. Synthetic metabolism: metabolic engineering meets enzyme design. Curr. Opin. Chem. Biol. 37, 56-62 (2017).

38. Hammer, S. C., Knight, A. M. \& Arnold, F. H. Design and evolution of enzymes for non-natural chemistry. Curr. Opin. Green Sustain. Chem. 7, 23-30 (2017).

39. Arnold, F. H. Directed evolution: bringing new chemistry to life. Angew. Chem. Int. Ed. 57, 4143-4148 (2018).

40. Guo, F. \& Berglund, P. Transaminase biocatalysis: optimization and application. Green Chem. 19, 333360 (2016).

41. $\mathrm{Fu}, \mathrm{H}$. et al. Chemoenzymatic asymmetric synthesis of the metallo- $\beta$-lactamase inhibitor aspergillomarasmine A and related aminocarboxylic acids. Nat. Catal. 1, 186-191 (2018).

42. Cui, Y. et al. Development of a versatile and efficient $\mathrm{C}-\mathrm{N}$ lyase platform for asymmetric hydroamination via computational enzyme redesign. Nat. Catal. 4, 364-373 (2021).

43. Roddan, R., Ward, J. M., Keep, N. H. \& Hailes, H. C. Pictet-Spenglerases in alkaloid biosynthesis: future applications in biocatalysis. Curr. Opin. Chem. Biol. 55, 69-76 (2020).

44. Brovetto, M., Gamenara, D., Méndez, P. S. \& Seoane, G. A. C-C bond-forming lyases in organic synthesis. Chem. Rev. 111, 4346-4403 (2011).

45. Leisch, H., Morley, K. \& Lau, P. C. K. Baeyer-Villiger monooxygenases: more than just green chemistry. Chem. Rev. 111, 4165-222 (2011).

46. Knaus, T., Böhmer, W. \& Mutti, F. G. Amine dehydrogenases: efficient biocatalysts for the reductive amination of carbonyl compounds. Green Chem. 19, 453-463 (2017).

47. Rudroff, F. et al. Opportunities and Challenges for for combining chemo- and biocatalysis. Nat. Catal. 1, 12-22 (2018).

48. Köhler, V. \& Turner, N. J. Artificial concurrent catalytic processes involving enzymes. Chem. Commun. 51, 450-464 (2015).

49. Mitschke, B., Turberg, M. \& List, B. Confinement as a Unifying Element in Selective Catalysis. Chem 6, 2515-2532 (2020). 